\title{
BIOMARCADORES PARA EL DIAGNÓSTICO DE INFECCIONES ARTICULARES PERIPROTÉSICAS
}

BIOMARKERS FOR THE DIAGNOSIS OF PERIPROSTHETIC JOINT INFECTIONS

\section{Jaime IBARROLA ${ }^{1}$.}

${ }^{1}$ Profesor Asistente de Ortopedia y Traumatología, Facultad de Ciencias Médicas, Universidad Nacional de Asunción, San Lorenzo - Paraguay.

Cómo citar este artículo: Ibarrola J. Biomarcadores para el diagnóstico de infecciones articulares periprotésicas. Medicina Clínica y Social. 2018;2(1):45-47.

\section{Estimado Editor,}

En los últimos años, la ciencia ortopédica ha evolucionado y ha iniciado un proceso de búsqueda de una única prueba de referencia estándar para determinar la existencia de infecciones articulares periprotésicas. Esto último se ha dado a través del análisis del líquido sinovial y del suero sanguíneo, con la identificación de numerosos biomarcadores como posibles candidatos (1).

¿Por qué este afán en encontrar una prueba única diagnóstica? Esto se debe, principalmente, a que la infección articular periprotésica después de una artroplastia total, ya sea de cadera o rodilla, es una complicación muy temida por los cirujanos ortopédicos, pues termina generando una evolución tórpida y negativa del paciente (en lo físico, psicosocial, emocional y económico) (2). De ahí la importancia del diagnóstico rápido y preciso de este tipo de complicación.

Hasta ahora, las pruebas convencionales para el diagnóstico de una infección articular periprotésica presentaban problemas de precisión y también de viabilidad (3), por lo que clínicos e investigadores se han volcado a la búsqueda de otras pruebas diagnósticas.

Además de los biomarcadores "convencionales" tales como la concentración sérica de proteína $C$ reactiva y el recuento de glóbulos blancos en el líquido sinovial, ahora se está descubriendo un número creciente de proteínas antimicrobianas y citoquinas proinflamatorias más específicas (3). Es sabido que, en la Medicina, los biomarcadores se utilizan con fines de diagnóstico y de pronóstico, ya que pueden determinar objetivamente la presencia de una enfermedad o un estado biológico particular. En ese sentido, en el área de la Ortopedia y la Traumatología, también cada vez hay más pruebas que apoyan la búsqueda y medición de biomarcadores específicos en suero y/o líquido sinovial de pacientes con sospecha de infecciones articulares (4). Los biomarcadores séricos y sinoviales no solo tienen el potencial de mejorar el diagnóstico de las infecciones articulares periprotésicas, sino que también pueden guiar al ortopedista y al especialista en infectología en la terapia antibiótica y en la respuesta al tratamiento quirúrgico.

La tabla 1 resume aquellos biomarcadores que actualmente cuentan con algún nivel de evidencia (5-8). 
TABLA 1. BIOMARCADORES EN INFECCIÓN ARTICULAR PERIPROTÉSICA

\begin{tabular}{|l|r|}
\hline Biomarcadores séricos & $\begin{array}{r}\text { IL-4; IL-6; TNF- } \alpha \text {; Procalcitonina; Molécula soluble } \\
\text { de adhesión intercelular } 1 \text { (sICAM-1); Dímero-D. }\end{array}$ \\
\hline Biomarcadores sinoviales & $\begin{array}{r}\text { Proteína C reactiva; Esterasa leucocitaria; } \beta \text {-defensina humana-2; } \beta- \\
\text { defensina humana-3; IL-1 } \beta ; \text { IL-6; IL-8; IL-17; TNF- } \alpha \text {; Interferón- } \delta \text { y fac- } \\
\text { tor de crecimiento del endotelial vascular. }\end{array}$ \\
\hline
\end{tabular}

De entre todos los biomarcadores citados en la tabla 1, el recuento de glóbulos blancos en el líquido sinovial, la proteína C reactiva, IL-6, IL-8 y la defensina humana son los que se muestran más promisorios para el diagnóstico preciso de infecciones articulares periprotésicas (1). No obstante, todavía no se cuenta con una única prueba lo suficientemente precisa, rentable y factible para el diagnóstico de este tipo de infecciones. Por lo tanto, y hasta que se encuentre esa "única prueba", los biomarcadores existentes deberán utilizarse en conjunto con otras pruebas diagnósticas convencionales para facilitar el diagnóstico y manejo de estas complicaciones en los pacientes.

\section{CONFLICTOS DE INTERÉS Y FUENTE DE FINANCIACIÓN}

El autor declara no poseer conflictos de interés. Fuente de financiación: ninguna.

\section{REFERENCIAS BIBLIOGRÁFICAS}

1. Lee YS, Koo KH, Kim HJ, Tian S, Kim TW, Maltenfort MG, et al. Synovial Fluid Biomarkers for the Diagnosis of Periprosthetic Joint Infection: A Systematic Review and Meta-Analysis. The Journal of Bone and Joint Surgery. 99(24):2077-2084. https://doi.org/10.2106/JBJS.17.00123

2. Parvizi J, Ghanem E, Azzam K, Davis E, Jaberi F, Hozack W. Periprosthetic infection: are current treatment strategies adequate? Acta Orthop Belg. 2008;74(6):793-800. URL.

3. Alvand A, Rezapoor M, Parvizi J. The Role of Biomarkers for the Diagnosis of ImplantRelated Infections in Orthopaedics and Trauma. Adv Exp Med Biol. 2017;971:69-79. https://doi.org/10.1007/5584 201711

4. Deirmengian C, Kardos K, Kilmartin P, Cameron A, Schiller K, Parvizi J. Diagnosing periprosthetic joint infection: has the era of the biomarker arrived? Clin Orthop Relat Res 2014;472(11):3254-3262. https://doi.org/10.1007/s11999-014-3543-8

5. Drago L, Vassena C, Dozio E, Corsi MM, De Vecchi E, Mattina R, et al. Procalcitonin, Creactive protein, interleukin- 6 , and soluble intercellular adhesion molecule-1 as markers of postoperative orthopaedic joint prosthesis infections. Int J Immunopathol Pharmacol 2011;24(2):433-440. https://doi.org/10.1177/039463201102400216

6. Ghanem E, Antoci V Jr, Pulido L, Joshi A, Hozack W, Parvizi J. The use of receiver operating characteristics analysis in determining erythrocyte sedimentation rate and C-reactive protein levels in diagnosing periprosthetic infection prior to revision total hip arthroplasty. Int J Infect Dis 2009;13(6):e444-e449. https://doi.org/10.1016/j.ijid.2009.02.017 
7. Ghanem E, Azzam K, Seeley M, Joshi A, Parvizi J. Staged revision for knee arthroplasty infection: what is the role of serologic tests before reimplantation? Clin Orthop Relat Res 2009;467(7):1699-1705. https://doi.org/10.1007/s11999-009-0742-9

8. Glehr M, Friesenbichler J, Hofmann G, Bernhardt GA, Zacherl M, Avian A, et al. Novel biomarkers to detect infection in revision hip and knee arthroplasties. Clin Orthop Relat Res 2013;471(8):2621-2628. https://doi.org/10.1007/s11999-013-2998-3 